\title{
The effect of environmental pollutants and food processing on the development of antibiotic resistance
}

\author{
Lucia Birosova ${ }^{a}$, Maria Mikulasovab
}

\begin{abstract}
Aim. The purpose of this study was to determine how various compounds known to be positive mutagens, contribute to the development of mutations leading to ciprofloxacin resistance in Salmonella enterica subsp. enterica serotype Typhimurium. The molecular mechanism of ciprofloxacin resistance in treated strains was investigated.

Methods. A modified version of the incorporation plate test was used for quantitative determination of ciprofloxacin resistant mutants and for assessment of the mutation frequency induced by the positive mutagens in different concentrations. An AS-PCR-RFLP for monitoring of gyrA mutations was applied.

Results. Mutation frequency, expressed as number of antibiotic resistant colonies per $10^{8}$ viable cells, was much higher after exposure of bacterial cells to 3-(5-nitro-2-furyl) acrylic acid and 2-nitrofluorene. All isolated cultures retain decreased susceptibility to antibiotic after multiple passages in antibiotic-free medium. 2-nitrofluorene was the best inducer of mutations in gyrA and in regulation genes affecting suppression of synthesis of outer membrane porins. 3-(5-nitro-2-furyl) acrylic acid gives rise to overproduction of efflux pump.
\end{abstract}

Conclusions. The data suggest that antibiotic resistance may not be only a consequence of misuse of antibiotics. A polluted environment as well as food processing could contribute to this unwanted process.

Key words: ciprofloxacin, mutagens, resistance, Salmonella

Received: December 9, 2011; Accepted with revision: June 25, 2012; Available online: October 31, 2012

http://dx.doi.org/10.5507/bp.2012.067

aDepartment of Nutrition and Food Assessment, Faculty of Chemical and Food Technology, Slovak University of Technology, Bratislava, Slovak Republic

${ }^{b}$ Department of Molecular Biology, Faculty of Natural Sciences, Comenius University, Bratislava, Slovak Republic

Corresponding author: Lucia Birosova, e-mail: lucia.birosova@stuba.sk

\section{INTRODUCTION}

The necessity to retain the efficiency of antibiotics and curtail the expansion of antibiotic resistance has already overwhelmed the scope of health care systems and has become a serious socioeconomic problem.

Bacterial resistance could be caused by horizontal gene transfer, recombination processes and also by genome mutation. Although antibiotic resistance has been studied intensively, mechanisms that generate resistance mutations are poorly understood. Chromosomal mutations can lead to antibiotic resistance in several different ways. In recent years, much attention has been given to the idea that the mutation process in bacterial populations is not a static event but a complex network of factors influencing the rate and the type of mutants ${ }^{1}$. Different stresses such as starvation, oxidation or UV irradiation can increase the mutation rates of bacteria ${ }^{2}$. All factors affecting the mutability of chromosomal genes confer resistance among different bacterial species ${ }^{3}$. As suggested by Martinez and Baquero ${ }^{1}$ mutation frequencies are probably much higher in the course of infection than those determined in vitro, because bacteria growing in vivo are frequently under environmental stress and challenges. Pathogenic bacteria are exposed to different chemical and physical agents which may damage DNA resulting in mutations.
Salmonella is one of the primary causes of bacterial foodborne infections in humans causing illnesses and fatalities all over the world each year. Some of these infections result in bacteraemia requiring treatment with antibiotics ${ }^{4}$. Fluoroquinolones have been used successfully to treat salmonellosis and are the commonly recommended agent of first choice but there is evidence of an increasing incidence of strains that exhibit resistance or decreased susceptibility to the most recent fluoroquinolones $^{5.8}$. Two mechanisms are involved in the development of resistance to fluoroquinolones: alterations in drug targets DNA gyrase or topoisomerase IV ( ref. $^{9,10}$ ) and decreased cellular accumulation of quinolones ${ }^{11}$. Mutations causing quinolone resistance occur primarily in a highly conserved region (QRDR) of DNA gyrase and topoisomerase IV genes ${ }^{12}$. Mutations affecting the accumulation of quinolones include those that affect the regulatory gene marA (multiple antibiotic resistance) (ref. ${ }^{13,14}$ ) generally cause decreased expression of the OmpF porin ${ }^{15}$ and overexpression of the AcrAB efflux pump ${ }^{16}$. These porin and pump changes lead to resistance not only to quinolones but also to a number of structurally unrelated compounds ${ }^{17}$.

The importance of de novo mutations in the development of antibiotic resistance prompted us to ask how compounds known as positive mutagens contribute to the development of this unwanted process. The aim of this paper was determine the frequency of induced mutations 
that may lead to antibiotic resistance as well as to determine the mechanism of ciprofloxacin resistance induced by tested positive mutagens.

\section{MATERIALS AND METHODS}

\section{Bacterial strains and drugs}

The bacterial strain used in this study Salmonella enterica, subs. enterica serotype Typhimurium CCM 4763 was received from the Collection of Microorganisms, Masaryk University, Brno (Czech Republic). Mutant strains were prepared. Staphylococcus aureus CCM 885 was obtained from the same collection.

Ciprofloxacin was purchased from Merck (Germany). 2-nitrofluorene (2NF, Sigma Aldrich, Germany) and 2-(5-nitro-2-furyl) acrylic acid (5NFAA, Slovakofarma, Slovak Republic) were dissolved in dimethylsulfoxid (DMSO, Merck, Germany)

\section{Antimicrobial susceptibility determination}

The minimal inhibition concentration (MIC) of antibiotic was determined by the agar dilution method using agar plates containing serial twofold dilutions of drug according to the Clinical and Laboratory Standards Institute guidelines (formerly the National Committee for Clinical Laboratory Standards) (ref. ${ }^{18}$ ), and by microsuspenssion assay in 96-well microplates ${ }^{19}$. The MIC was defined as the lowest concentration of drug that inhibits visible growth after $24 \mathrm{~h}$ of incubation at $37^{\circ} \mathrm{C}$. The MICs reported here represent the arithmetic mean of the means of three separate experiments.

\section{Determination of mutation frequencies to antibiotic resistance}

S. enterica ser. Typhimurium strain was grown overnight in antibiotic-free broth with viable cell number around $10^{9} \mathrm{~mL}^{-1}$. This culture was divided into $0.1 \mathrm{~mL}$ aliquots, then $0.1 \mathrm{~mL}$ of positive mutagen and $0.5 \mathrm{~mL}$ of phosphate buffer of $\mathrm{pH} 7.4$ was added and cultures were treated with mutagen for $30 \mathrm{~min}$. Later the fresh Luria-Bertani (LB) medium was added and cultures were incubated for $3 \mathrm{~h}$ at $37{ }^{\circ} \mathrm{C}$ to allow a few cell divisions and protein expression to occur. The number of ciprofloxacin-resistant mutants that emerged in each culture was determined by plating the entire culture on LB agar plates containing a selective concentration of antibiotic. The total number of viable cells was determined by plating an appropriate dilution of cultures on nonselective medium. Colonies on both selective and nonselective plates were counted after incubation for 2 days. The frequency of resistant mutants (resistance index RI) was expressed as mean number of resistant cells divided by the total number of viable cells per culture. RI factor represents proportion of induced mutation frequency to frequency of spontaneous mutations leading to ciprofloxacin resistance. Data shown here represent the mean of three independent experiments; each experiment was designed in five parallels and statistically evaluated by the Student's t-test.

\section{Subculturing of mutant isolates}

From each of 50 cultures growing in selective antibiotic concentration one random mutant colony was picked and passaged in media without antibiotics for ten consecutive days. Following serial culture, the ciprofloxacin MIC of cells collected after the first and last subculture to antibiotics was determined. Strains, which maintained increased values of MIC, were considered to be resistant mutants. The cells from the same colonies on selective plates were also transferred onto medium containing twice of selective concentration of the antibiotic. After $24 \mathrm{~h}$ of incubation, surviving cells were transferred onto medium containing twice the prior concentration of ciprofloxacin and this process was repeated serially until no growth occurred.

\section{Detection of gyrA mutations by AS-PCR-RFLP assay}

AS-PCR-RFLP (allele specific - PCR - restriction fragment length polymorphism) assay was provided according Giraud et al. ${ }^{20}$. The sequences of all primers used in PCR amplification are shown in Table 1. The forward primer (STGYRA1) and reverse primer (STGYRAHinfl/87) were predicted to produce a 195 bp fragment with a Hinfl restriction site for determination of mutations in codon 83 and 87 and allele-specific forward primer (AS-81) for determination of mutation on the 81st codon. PCR was performed with hot-start technique in total volume of $50 \mu \mathrm{L}$, which contained 25 pmol of primers STGYRA1 and AS-81, 50 pmol of primer STGYRAHinfl/87, $200 \mu \mathrm{M}$ deoxynucleotide triphosphates, $2 \mathrm{mM}$ $\mathrm{MgCl}_{2}$ and $0.5 \mathrm{U}$ of Taq polymerase (Promega, Madison). After a denaturation step of 15 min at $95^{\circ} \mathrm{C}$ (denaturation of bacterial colonies) denaturation of $3 \mathrm{~min}$ at $94{ }^{\circ} \mathrm{C}$ was performed, followed by amplification performed over 30 cycles, each one consisting of $1 \mathrm{~min}$ at $94{ }^{\circ} \mathrm{C}, 1 \mathrm{~min}$ hybridization temperature at $55^{\circ} \mathrm{C}$ and $1 \mathrm{~min}$ at $72{ }^{\circ} \mathrm{C}$, with a final extension step of $10 \mathrm{~min}$ at $72{ }^{\circ} \mathrm{C}$. Primers and free nucleotides were removed with a Qiaquick spin PCR purification kit (Quiagen S.A., France). Restriction enzyme

Table 1. Primers used for PCR amplification and sequencing.

\begin{tabular}{ll}
\hline Primer & Sequence \\
\hline STGYRA1 & 5'-TGTCCGAGATGGCCTGAAGC-3' \\
STGYRA12 & 5'-CGTTGATGACTTCCGTCAG-3' \\
STGYRA-HinfI/87 & 5'-ATGTAACGCAGCGAGAATGGCTGCGCCATACGAACGATGGAG-3' \\
AS-81 & 5'-GGTAAATACCATCCCCACG-3' \\
\hline
\end{tabular}


digestion was performed in $10 \mu \mathrm{L}$ mixture containing 5 $\mu \mathrm{L}$ of PCR product and $5 \mathrm{U}$ of Hinfl. Both digested and undigested PCR products were resolved in 3\% (w/v) agarose gel for small fragments. DNA ladder (25 - $500 \mathrm{bp}$ ) was used as a molecular marker. A PCR product prepared with 2 primers (STGYRA1 and STGYRA2) was used for sequence determination and PCR reaction was performed as described for AS-PCR-RFLP.

\section{Preparation and analysis of outer-membrane protein (OMP)}

Expression of OmpF was determined according Giraud et al. ${ }^{21}$ with slight modification. Briefly, bacteria were mechanically disrupted with glass beads, unbroken cells were removed by centrifugation and supernatant was incubated with $1 \%(\mathrm{v} / \mathrm{v})$ Triton X-100 and 2\% (v/v) lauroyl sarcosine. After ultracentrifugation SDS-PAGE was performed and proteins were detected after staining with Coomassie Brilliant Blue.

\section{Measurement of ciprofloxacin accumulation}

The uptake of ciprofloxacin by bacterial cells was measured according to Hirai et al. ${ }^{22}$. Ciprofloxacin at a final concentration of $10 \mu \mathrm{g} \mathrm{mL} \mathrm{L}^{-1}$ was added to the bacterial cell suspension $\left(\mathrm{A}_{570}=0.7\right)$, and the cultures were incubated at $37{ }^{\circ} \mathrm{C}$ with shaking. In the indicated times $(0,5,10$, $15,25,35 \mathrm{~min}), 10 \mathrm{~mL}$ of the culture was chilled and the cells were sedimented by centrifugation. The cells were then suspended in $1 \mathrm{~mL}$ of saline. The suspension was immersed in boiling water for $10 \mathrm{~min}$ and then centrifuged. All drugs could be extracted from the cells by this treatment and it was also confirmed that ciprofloxacin was not inactivated by boiling. The concentration of ciprofloxacin in the supernatants was measured by bioassay using Staphylococcus aureus ${ }^{23}$. The inhibition zones produced by $20 \mu \mathrm{L}$ aliquots of each extract were compared with those produced by $20 \mu \mathrm{L}$ aliquots of different known ciprofloxacin concentrations using nonlinear regression. The accepted standard deviation for all ciprofloxacin uptake results was always $\leq 5 \%$ with respect to each mean value of three measurements. Likewise, all the above were assayed in the presence of $30 \mu \mathrm{M} 3,3^{\prime}, 4^{\prime}, 5$-tetrachlorosalicylanilide (TCS), an uncoupler, added in the $10^{\text {th }}$ minute. For confirmation, ciprofloxacin concentrations were also measured by HPLC.

\section{RESULTS}

Mutation frequency and susceptibility of isolated mutants

The mutagenic effect of $2 \mathrm{NF}$ and 5NFAA in low concentrations is shown in Fig.1. The frequency of mutations leading to ciprofloxacin resistance was increased significantly with increasing concentration of mutagen. In comparison, 5NFAA was a better inducer of ciprofloxacin resistance because the mutation frequency increased up to 400 -fold. For $2 \mathrm{NF}$ it was only 8 -fold over spontaneous background levels with increasing concentrations of mutagen.
After 10 passages in antibiotic-free medium, all isolated strains were able to grow on selective plates with ciprofloxacin. The sensitivity of these strains to ciprofloxacin decreased very moderately; the majority of strains had ciprofloxacin MIC of $0.78 \mu \mathrm{g} \mathrm{mL}^{-1}$ and only $20 \%$ of 5NFAA induced mutants were resistant. Some strains had adapted to higher ciprofloxacin concentrations after the serial passaging on medium with increasing antibiotic concentration. The sensitivity to ciprofloxacin had decreased; even in $35 \%$ of strains (for both mutagenic compounds) we observed a very high, clinically significant value MIC of ciprofloxacin $\left(25\right.$ - $\left.200 \mu \mathrm{g} \cdot \mathrm{mL}^{-1}\right)$. According to National Antimicrobial Susceptibility Program ${ }^{24}$, ciprofloxacin susceptibility limit for Salmonella Typhimurium is $4 \mu \mathrm{g} . \mathrm{mL}^{-1}$, and bacteria which grow in the presence of ciprofloxacin in these and higher concentration are considered to be resistant.

\section{Mutations in gyrA gene}

For determination of mutations in the gyrA gene associated with quinolone resistance, all strains with ability to grow on selective concentration of ciprofloxacin were examined for mutations at Gly-81, Ser-83 and Asp-87 in the gyrA gene by the AS-PCR-RFLP assay according to Giraud et al. ${ }^{18}$. Fig. 2 presents representative agarose gel electrophoresis profiles obtained in the assay with the parent strain and resistant isolates.

All 2NF - mutants had a mutation in the QRDR of gyrA gene. All resistant strains (MIC $>4 \mu \mathrm{g} \cdot \mathrm{mL}^{-1}$ ) had mutation in codon Ser-83 while strains with decreased susceptibility had mutation in codon Asp-87 or Ser-83. In $90 \%$ of strains generated after 5NFAA effect we determined mutation only in Ser-83 and no other type of mutation was observed.

DNA sequencing of the $500 \mathrm{bp}$ PCR products covering the entire QRDR of gyrA of all mutants with mutations in either of these codons revealed base substitutions in the codon Ser-83 from TCC (Ser) to TTC (Phe) and in the codon Asp-87 from GAC (Asp) to AAC (Asn).

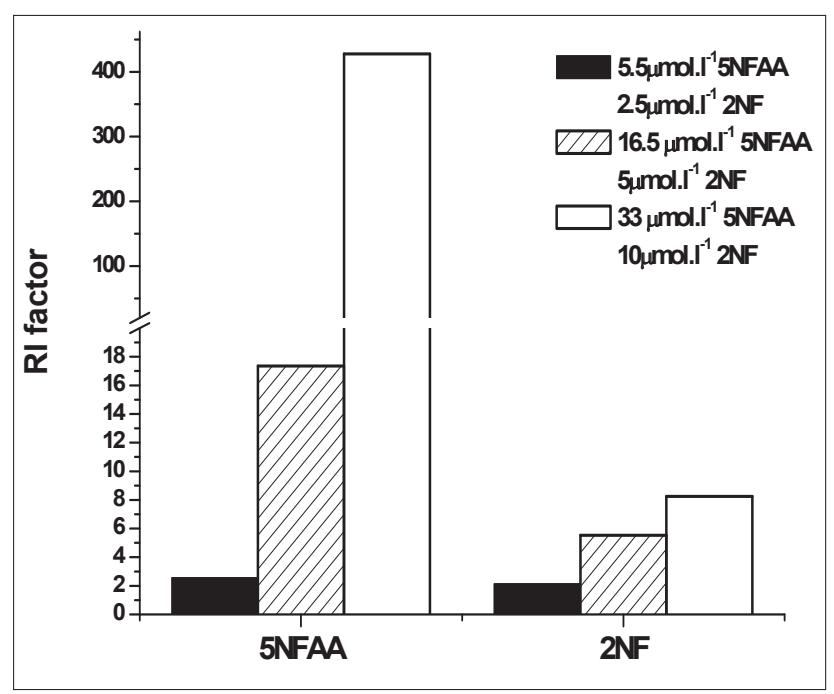

Fig. 1. Effect of 2-(5-nitro-2-furyl) acrylic acid and 2-nitrofluorene on frequency of mutations leading to ciprofloxacin resistance. 


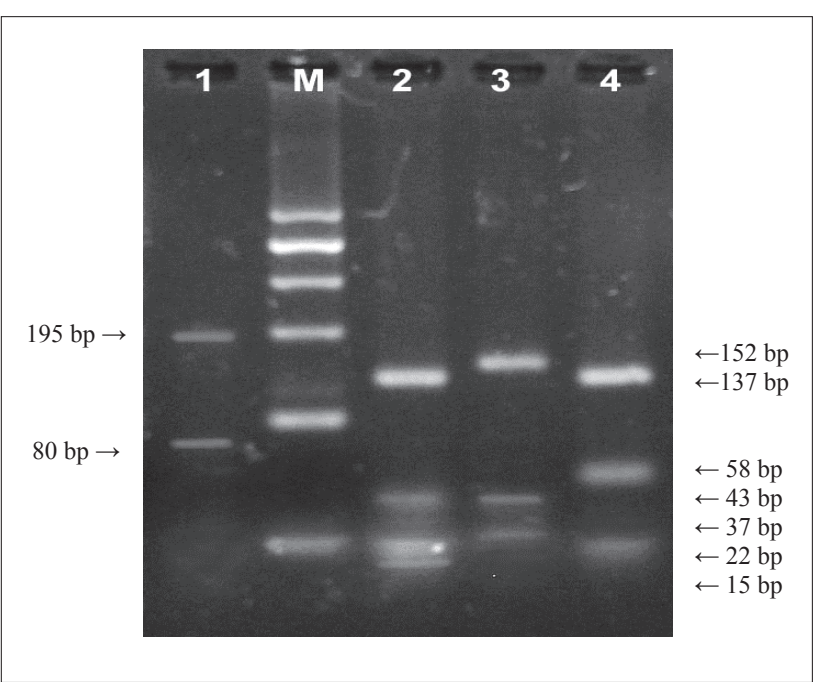

Fig. 2. AS-PCR-RFLP patterns of representative strains before HinfI digestion (1) and after digestion without mutation (2), with mutation in 83 codon (3) and in 87 codon (4), M - DNA marker (25-500 bp).

We found no Gly-81 mutation nor the combination of the mentioned mutations. It is remarkable that mostly mutation in the 83rd codon led to clinically significant values of ciprofloxacin MIC.

\section{Changes in ciprofloxacin uptake}

The same strains were analyzed considering $\mathrm{OmpF}$ porin production and the efflux pump expression to determine changed antibiotic concentration in cells. We discovered that $\mathrm{OmpF}$ proteins were significantly reduced only in the case of $2 \mathrm{NF}$ mutant strains. Decreased levels or total absence of $\mathrm{OmpF}$ was determined in $88 \%$ of isolated strains (Fig. 3, lane 4,5). In strains affected by 5NFAA we

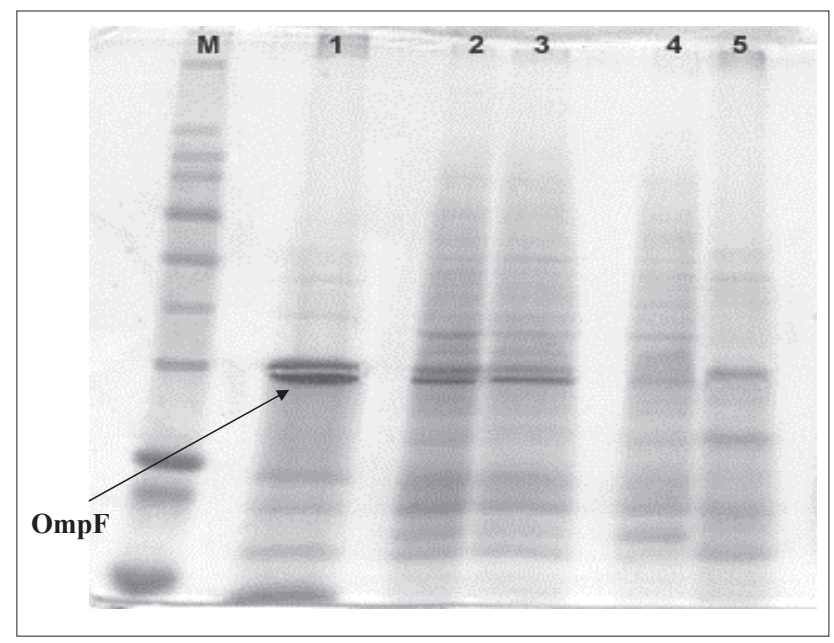

Fig. 3. OMP profiles prepared from parent strain (lane 1), 5NFAA mutant strains with decreased expression OmpF levels (lanes 2,3) and 2NF mutant strains with decreased levels or absence of OmpF (lane 4, 5). M - Sigma Marker ${ }^{\mathrm{TM}}$ Wide molecular weight range $(6,2-205 \mathrm{kDa})$. detected reduced levels of $\mathrm{OmpF}$ only in $33 \%$ of strains, all rest mutants had levels of $\mathrm{OmpF}$ proteins equivalent to parent, ciprofloxacin susceptible strain.

Fig. 4 shows the uptake of ciprofloxacin by parent strain and mutant NF12 (2NF induced mutant) in which increased efflux was detected. Mutant strain accumulated approximately $50 \%$ less ciprofloxacin than parent strain. After addition of an energy inhibitor TCS to the cultures, accumulation of ciprofloxacin increased rapidly in both strains. Uptake increase after TCS addition indicated blockage of an energy-dependent ciprofloxacin efflux.

Such course with minor differences was found in $66 \%$ of strains generated after exposure to 5NFAA, even in half of them we registered simultaneously decreased levels of OmpF and these entire mutants were resistant to ciprofloxacin. Only $20 \%$ of 2 NF mutants had efflux pump overproduction. Simultaneously absence of porins was detected. All these strains had only decreased susceptibility to ciprofloxacin.

\section{DISCUSSION}

Salmonella Typhimurium is a foodborne pathogen which colonizes the intestine where it may make contact with mutagens present in some food-stuffs or generated during food processing. Accordingly we focused on the effect of two positive mutagens on the development of ciprofloxacin resistance in Salmonella enterica subs. enterica serotype Typhimurium. 2-Nitrofluorene is a typical carcinogenic nitropolycyclic aromatic hydrocarbon ${ }^{25,26}$ which was detected in diesel exhaust particles ${ }^{27}$ and also as a major pollutant in the atmosphere ${ }^{28}$. In our previous study we discovered that this compound induces mutations leading to antibiotic resistance and selects for high level resistant strains. 2-(5-nitro-2-furyl) acrylic acid used to be a wine stabilizer ${ }^{29}$.

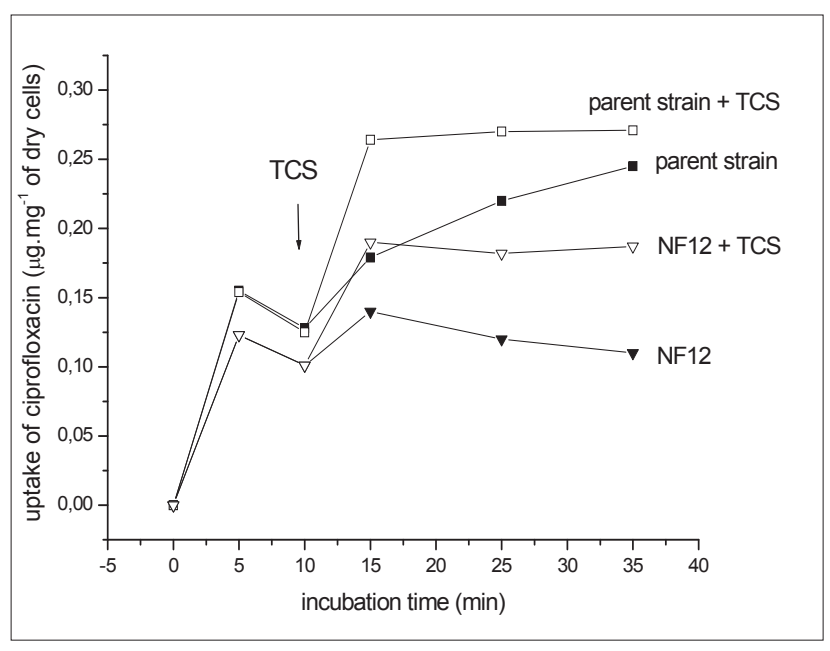

Fig. 4. Uptake of ciprofloxacin by parent strain and resistant mutant NF12 generated after 2NF exposition with and without addition of TCS. 
Positive mutagens used in this study differ in the induction of mutations leading to ciprofloxacin resistance. We also found that doses of mutagens effective in the Ames test did not have to correlate with that effective for antibiotic resistance development. 5NFAA in lower concentrations increased the frequency of mutations leading to ciprofloxacin resistance at a higher rate than $2 \mathrm{NF}$.

Mutations at Asp- 87 or Ser-83 in the quinolone resistance-determining region of the $g y r A$ gene were found in all mutants isolated after $2 \mathrm{NF}$ induction. Although mutations at Asp-87 have usually been reported to be concomitant with mutations at Ser-83 (ref. ${ }^{30}$ ), we found a single mutation at Asp- 87 in $60 \%$ of $2 \mathrm{NF}$ mutants in gyrase gene. Nor after adaptation did we obtain mutant strains with double mutation in Ser-83 and Asp-87 in gyrA. In 5NFAA mutants we detected only one type of point mutation at Ser-83 of the gyrA.

Mutations in regulatory genes of MarRAB operon, which regulate expression of efflux pumps and outer membrane porins can also contribute to high-level fluoroquinolone resistance in Salmonella Typhimurium ${ }^{31}$. We discovered that mutation at the 83rd codon of the gyrA together with absence of OmpF was always connected with the development of ciprofloxacin resistance. 5NFAA was the best inductor of efflux pump overproduction; $2 \mathrm{NF}$ was very effective in repression of OmpF synthesis.

The link between mutagenicity and a clinical parameter such as antibiotic resistance would provide valuable data to help us understand the problem of the development of drug resistance as well as that associated with the interpretation of mutagenic studies. Knowledge of the effects of some environmental factors on the mutation rate can enable us to predict how fast the bacteria will develop antibiotic resistance in a given environment.

\section{CONCLUSIONS}

Many studies indicate that the main cause of antibiotic resistance of pathogenic bacteria is overuse or misuse of antibiotics in human and veterinary medicine. Our results show that environmental pollutants as well as compounds generated during food processing may contribute to the development of mutations leading to this unwanted process. Both tested compounds significantly increased mutation frequency leading to ciprofloxacin resistance in Salmonella Typhimurium. 2-nitrofluorene was the best inducer of mutations in gyrA and in regulation genes affecting synthesis inhibition of outer membrane porins. 3-(5-nitro-2-furyl) acrylic acid gave rise to overproduction of efflux pump.

\section{REFERENCES}

1. Martinez JL, Baquero F. Mutation frequencies and antibiotic resistance. Antimicrob Agents Chemother 2000;44:1771-7. doi: 10.1128/ AAC.44.7.1771-1777.2000

2. Tenaillon O, Denamur E, Matic I. Evolutionary significance of stressinduced mutagenesis in bacteria. TRENDS Microbiol 2004;12:264-70. doi:10.1016/j.tim.2004.04.002
3. Monteiro ACM, Ferreira RCC, Padilla G, Ferreira LCS, Costa SOP. Environmental and genetic factors affecting mutabillity to aminoglycoside antibiotics among Escherichia coli K12 strains. Gen Mol Biol 2003;26:221-7. doi: 10.1590/S1415-47572003000200017

4. Mead PS, Slutsker L, Dietz V, McCaig LF, Bresee JS, Shapiro C, Griffin PM, Tauxe RV. Food-related illnesses and death in the United States. Emerg Infect Dis 1999;5:607-25.

5. Chen S, Cui S, McDermott PF, Zhao S, White DG, Paulsen I, Meng J. Contribution of target gene mutations and efflux to decreased susceptibility of Salmonella enterica serovar Typhimurium to fluoroquinolones and other antimicrobials. Antimicrob Agents Chemother 2007;51(2):535-42. doi: 10.1128/AAC.00600-06

6. Esaki H, Chiu ChH, Kojima A, Ishihara K, Asai T, Tamura Y, Watanabe $\mathrm{H}$. Comparison of fluoroquinolone resistance gene of Salmonella enterica serovar Choleraesuis isolates in Japan and Taiwan. Jpn J Infect Dis 2004;57:287-8.

7. Hopkins KL, Davies RH, Threlfall EJ. Mechanism of quinolone resistance in Escherichia coli and Salmonella. Int J Antimicrob Agents 2005;25:358-73. doi:10.1016/j.ijantimicag.2005.02.006

8. Harish BN, Menezes GA, Sarangapari K, Parija SC. Fluoroquinolone resistance among Salmonella enterica serovar Paratyphi A in Pondicherry. Indian J Med Res 2006;124:585-7.

9. Reyna F, Huesca M, Gonzalez V, Fuchs LY. Salmonella typhimurium gyrA mutations associated with fluoroquinolone resistance. Antimicrob Agents Chemother 1995;39:1621-3.

10. Brown JC, Thomson CJ, Aymes SGB. Mutations of the gyrA gene of clinical isolates of Salmonella typhimurium and three other Salmonella species leading to decreased susceptibilities to 4-quinolone drugs. J Antimicrob Chemother 1996;37:351-6.

11. Oethinger M, Kern V, Jellen-Ritter AS, McMurry LM, Levy SB. Ineffectiveness of topoisomerase mutations in mediating clinically significant fluoroquinolone resistance in Escherichia coli in the absence of the AcrAB efflux pump. Antimicrob Agents Chemother 2000;44:10-3.

12. Heisig P. Genetic evidence for a role of parC mutation in the development of high-level fluoroquinolone resistance in Escherichia coli. Antimicrob Agents Chemother 1996;40:879-85.

13. Cohen SP, Hächler H, Levy SB. Genetic and functional analysis of the multiple antibiotic resistance (mar) locus in Escherichia coli. J Bacteriol 1993;175:1484-92.

14. Randall LP, Woodward MJ. Multiple antibiotic resistance (mar) locus in Salmonella enterica serovar typhimurium DT104. Appl Environ Microbiol 2001;67:1190-7. doi: 10.1128/AEM.67.3.1190-1197.2001.

15. Chou JH, Greenberg JT, Demple B. Posttranscriptional repression of Escherichia coli $\mathrm{OmpF}$ protein in response to redox stress: positive control of the micF antisense RNA by the soxRS locus. J Bacteriol 1993;175:1026-31.

16. Escribano I, Rodriguez JC, Cebrian L, Royo G. The importance of active efflux systems in the quinolone resistance of clinical isolates of Salmonella spp. Int J Antimicrob Agents 2004;24:428-32. doi:10.1016/j.jjantimicag.2004.05.004.

17. Piddock LJV, White DG, Gensberg K, Pumbwe L, Griggs DJ. Evidence for an efflux pump mediating multiple antibiotic resistance in Salmonella enterica serovar Typhimurium. Antimicrob Agents Chemother 2000;44:3118-21.

18. NCCLS. Performance Standards for Antimicrobial Susceptibility Testing. 15th Informational Supplement M100-S15. Wayne PA: National Committee for Clinical Laboratory Standards; 2005.

19. Mikulášová M, Bohovicová I. Genotoxic effect of vanillin derivatives. Biologia 2000;55:229-34.

20. Giraud E, Brisabois A, Martel JL, Chaslus-Dancla E. Comparative Studies of Mutations in Animal Isolates and Experimental In Vitro- and In Vivo-Selected Mutants of Salmonella spp. Suggest a Counterselection of Highly Fluoroquinolone-Resistant Strains in the Field. Antimicrob Agents Chemother 1999;43:2131-7.

21. Giraud E, Cloeckaert A, Kerboeuf D, Chaslus-Dancla E. Evidence for active efflux as primary mechanism of resistance to ciprofloxacin in Salmonella enterica serovar Typhimurium. Antimicrob Agents Chemother 2000;44:1223-8. doi: 10.1128/AAC.44.5.1223-1228.2000.

22. Hirai K, Aoyama H, Suzue S, Irikura T, Iyobe S, Mitsuhashi S. Isolation and characterization of norfloxacin-resistant mutants of Escherichia coli K-12. Antimicrob Agents Chemother 1986;30:248-53.

23. Tavío MM, Villa J, Ruiz J, Martín-Sánchez AM, Jiménez de Anta MT. Mechanisms involved in the development of resistance to fluoro- 
quinolones in Escherichia coli isolates. J Antimicrob Chemother 1999;44:735-42. doi: 10.1093/jac/44.6.735.

24. Fedorka-Cray PJ, Miller M, Tollefson L, Dargatz DA, Wineland NE. National Antimicrobial Susceptibility Monitoring Program Veterinary isolates, United States Government Printing, Washington DC; 1998.

25. Miller JA, Sandin RB, Miller EC, Rusch HP. The carcinogenicity of compounds related to 2 -acetylaminofluorene. II. Variations in the bridges and the 2-substituent. Cancer Res 1995;15:188-99.

26. Möller L, Torndal UB, Eriksson LC, Gustafsson JÅ. The air pollutant 2-nitrofluorene as initiator and promoter in a liver model for chemical carcinogenesis. Carcinogenesis 1989;10:435-40.

27. Campbell RM, Milton LL. Capillary column gas chromatographic determination of nitro polycyclic aromatic compounds in particulate extracts. Anal Chem 1984;56:1026-30.
28. Beije B., Möller L. 2-Nitrofluorene and related compounds: prevalence and biological effects. Mutat Res 1988;196:177-209.

29. Ebringer L, Goráková K, Blasko M, Lagitová N, Halupa I. Determination of the genetic toxicity of 5-nitrofurylacrylic acid. Tsitol Genet 1986;20:377-8.

30. Conrad S, Oethinger M, Kaifel K, Klotz R, Marre R, Kera EV. gyrA mutations in high-level fluoroquinolone-resistant clinical isolates of Escherichia coli. J Antimicrob Chemother 1996;38:443-55.

31. Baucheron S, Shaun T, Boyd D, Mulvey MR, Chaslus-Dancla E, Cloeckaert A. AcrAB-TolC directs efflux-mediated multidrug resistance in Salmonella enterica serovar Typhimurium DT104. Antimicrob Agents Chemother 2004;48(10):3729-35. doi: 10.1128/ AAC.48.10.3729-3735.2004 